\title{
The stability of toroidal fields in stars
}

\author{
J. Braithwaite
}

\author{
Max-Planck-Institut für Astrophysik, Karl-Schwarzschild-Straße 1, Postfach 1317, 85741 Garching, Germany \\ e-mail: jon@mpa-garching.mpg.de
}

Received 12 May 2004 / Accepted 21 March 2006

ABSTRACT

\begin{abstract}
We present numerical models of hydromagnetic instabilities under the conditions prevailing in a stably stratified, non-convective stellar interior, and compare them with previous results of analytic work on instabilities in purely toroidal fields. We confirm that an $m=1$ mode ("kink") is the dominant instability in a toroidal field in which the field strength is proportional to distance from the axis, such as the field formed by the winding up of a weak field by differential rotation. We measure the growth rate of the instability as a function of field strength and rotation rate $\Omega$, and investigate the effects of a stabilising thermal stratification as well as magnetic and thermal diffusion on the stability. Where comparison is computationally feasible, the results agree with analytic predictions.
\end{abstract}

Key words. instabilities - magnetohydrodynamics (MHD) - stars: magnetic fields

\section{Introduction}

Magnetic fields probably play a significant role in the internal rotation of stars. Even a relatively weak magnetic field is sufficient to couple different parts of the star and maintain a state of nearly uniform rotation. For the interior of the present Sun, for example, a field of less than 1 Gauss would be able to transmit the torque exerted by the solar wind through the interior (Mestel 1953). The observed rotation in the core of the Sun (Chaplin et al. 2001) is quite uniform, suggesting that a magnetic field of this order or larger may actually be present. The progenitors of white dwarfs and supernovae go through giant stages in which the envelope rotates very slowly. The degree of coupling between core and envelope by a magnetic field in this stage will determine whether the rotation rates of pulsars and white dwarfs are just a remnant of the initial rotation of their progenitors, or if a secondary process must be responsible (Spruit \& Phinney 1998; Spruit 1998).

Models of gamma-ray bursts in which the central engine derives from the rapidly spinning core of a massive star (Woosley 1993; Paczyński 1998) also depend on the ability of the core to keep its high angular momentum for a sufficient period of time, in the face of magnetic spindown torques exerted by the slowly rotating envelope (Heger et al. 2000).

The uniform rotation of the solar core may be due to a magnetic field, but this field's origin, configuration and strength are not known. By analogy with the magnetic A-stars, one might speculate that a "fossil" magnetic field could exist in the core of the Sun (Cowling 1945; Braithwaite \& Spruit 2004). Since no net field is seen at the surface (averaged over the solar cycle), the radial component of such a fossil would however have to be weak - of the order of a Gauss or less. A field weaker than about this $1 \mathrm{G}$ will quickly wrap up into a predominantly toroidal field, under the action of the remaining (weak) differential rotation in the core.

The predominantly toroidal magnetic field resulting from this process will not increase in strength arbitrarily. Eventually, the energy density in the field will become large enough that a magnetic instability will set in.
Analytic work, (e.g. Tayler 1973), shows that any purely toroidal field should be unstable to instabilities on the magnetic axis of the star (pinch-type instabilities, under the influence of the strongly stabilising stratification in a radiative stellar interior, or "Tayler instabilities" hereafter). The growth rates of these instabilities are expected to be of the order of the time taken for an Alfvén wave to travel around the star on a toroidal field line. This is very short compared to the evolutionary timescale of the star. In a star like the Sun, for example, with a field of 1000 Gauss, the growth timescale $r \sqrt{4 \pi \rho} / B$ would be of the order of years, if $r=R_{\odot} / 2$ is taken, and $\rho=1.3 \mathrm{~g} / \mathrm{cm}^{3}$.

A magnetic field of this type can also be subject to other instabilities, such as the magnetic buoyancy (Parker 1955; Gilman 1970; Acheson 1978) and magnetic shear instabilities (Velikhov 1959; Acheson 1978; Balbus \& Hawley 1992; Menou et al. 2004). As was shown by Spruit (1999), the Tayler instability will be the first to appear as the strength of the toroidal field is increased. This is because with the magnetic buoyancy instability, as with all instabilities where displacements in the vertical are necessary, the stratification provides a strong stabilising force. The same is the case with the magnetic shear instability, whose effect in a stellar interior is very limited in comparison to its effect in accretion discs. In contrast, the Tayler instability occurs on the magnetic axis, where the magnetic field is perpendicular to gravity and the displacements caused by the instability are also perpendicular to gravity.

In this paper we aim to test numerically the instability mechanism, and to verify that the predictions of the analytic work are relevant: that they cover all instabilities actually present in a system consisting of a predominantly toroidal field in a stable stratification. Much of the analytical stability analyses have been done under a local approximation. This can be shown to be exact for the case of adiabatic instabilities in a non-rotating star, but not for the more interesting cases in which rotation and the effects of magnetic and thermal diffusion are taken into account. Though it is not expected that major instabilities have been missed, numerical simulations can provide an important check. 
It is much less certain how the magnetic field evolves once instability has set in. In a scenario developed by Spruit (2002), it is argued that the instability will lead to self-sustained dynamo action. The field remains predominantly toroidal, subject to decay by Tayler instability, but is continuously regenerated by the winding-up of irregularities produced by the instability. This scenario has been applied in stellar evolution calculations of the internal rotation of massive stars by Heger et al. (2003) and Maeder \& Meynet (2003).

The balance between wrapping-up by differential rotation on the one hand and the destruction of the toroidal field by Tayler instabilities on the other determines the strength and configuration of the field, and the the rate at which it transports angular momentum through the star.

The long-term goal is therefore to investigate the non-linear development of the instability. With 3-D numerical simulations we can determine how quickly an initial toroidal field decays (by reconnection across the magnetic axis), and to determine the type of magnetic field that is maintained by differential rotation in a stably stratified star, under the action of magnetic instabilities, and to develop from this a quantitative theory for the angular momentum transport by magnetic fields in stars. This is beyond the scope of this paper, but was looked at by Braithwaite (2006), where the operation of this differential-rotation driven magnetic dynamo was demonstrated.

\section{The nature of the instabilities}

We first consider adiabatic instabilities, that is, ignoring the effects of viscosity and of thermal and magnetic diffusion. The instabilities of a magnetic field in a stable stratification then depend on three parameters: the field strength, some measure of the stability of the stratification, and the rotation rate of the star. For stars rotating well below their critical (maximal) rate, and for the expected relatively low field strengths, the relative strength of the parameters is expressed by the ordering

$N \gg \Omega \quad$ and $\quad N \gg \Omega_{\mathrm{A}}$,

where $N$ is the buoyancy frequency, $\Omega$ is the rotational velocity, and $\Omega_{\mathrm{A}}$ is the Alfvén frequency given by $v_{\mathrm{A}} / R=B /(R \sqrt{4 \pi \rho})$, $R$ is the radius of the star and $v_{\mathrm{A}}$ is the Alfvén speed. This is the same, in effect, as saying that the thermal energy density is much greater than both the rotational and the magnetic energy densities.

We consider an azimuthal field $\boldsymbol{B}=\boldsymbol{e}_{\phi} B(\varpi)$. We define

$p \equiv \frac{\partial \ln B}{\partial \ln \varpi} \quad$ as $\quad \varpi \rightarrow 0$.

In cylindrical coordinates $(\varpi, \phi, z)$ where the $z$ axis is parallel to the magnetic axis, the displacements from equilibrium are of the form

$\xi \sim \mathrm{e}^{\mathrm{i}(l \varpi+m \phi+n z)+\sigma t}$.

The shape of the unstable displacements is shown in Fig. 1, for modes with $m=0,1,2$. The $m=1$ mode is known as the "kink" instability, and is seen in twisted solar coronal loops and twisted rubber bands. This instability sets in once the magnetic field component along the flux tube (vertical in the geometry used here) falls below some critical value in relation to the toroidal component. In our case of a predominantly toroidal field, resulting from winding up by differential rotation, the vertical component is neglected: it is a limiting case.
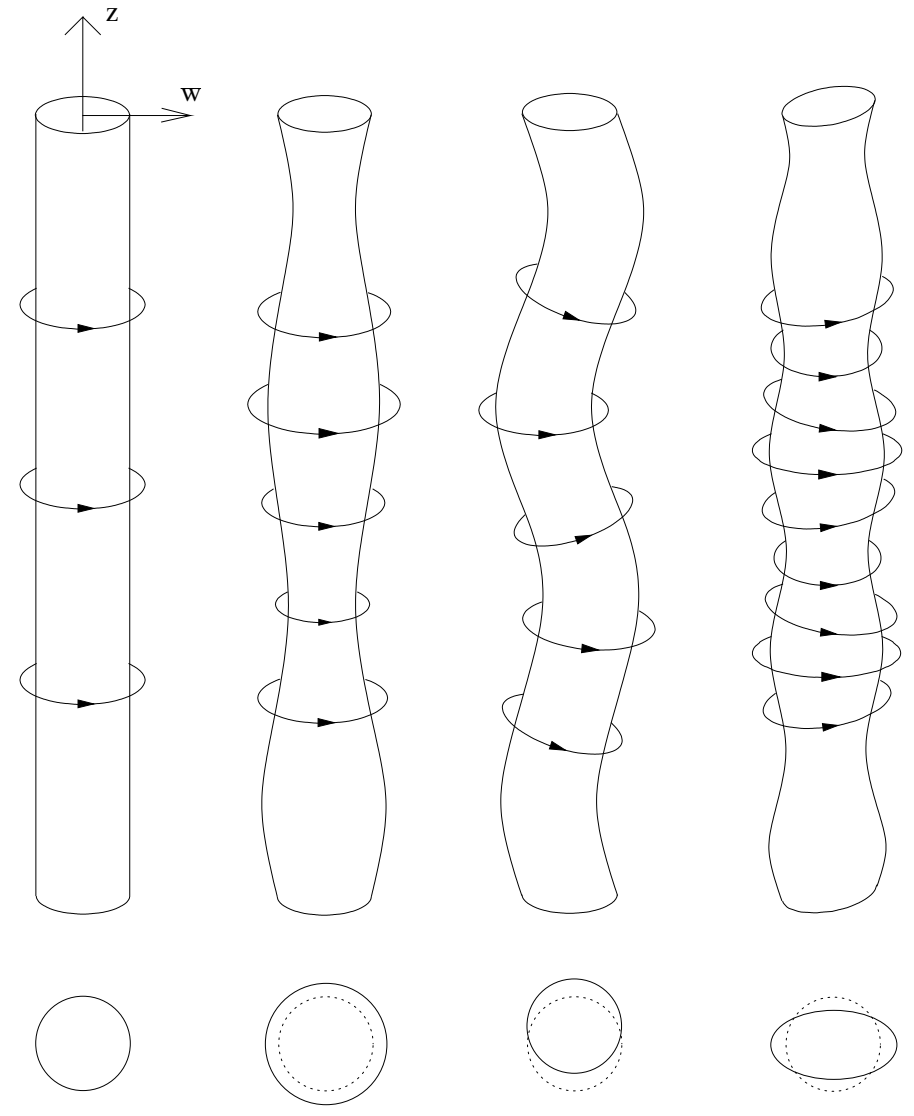

equilibrium

$$
\mathrm{m}=0
$$

$\mathrm{m}=1$

$\mathrm{m}=2$

Fig. 1. The physical form of the instability, for modes $m=0, m=1$ and $m=2$. Above, co-moving surfaces are drawn, with the magnetic field represented by the lines with arrows. The $z$ and $\varpi$ axes are marked on the diagram on the left; gravity (if present) acts along the $z$ axis. Below, cross-sections of these co-moving surfaces are plotted, with the dotted circles representing equilibrium.

It is predicted that for an instability to occur, we must have (Tayler 1957)

$$
p>\frac{m^{2}}{2}-1 \quad(m \neq 0) \quad \text { and } \quad p>1 \quad(m=0) .
$$

It is now useful to consider the likely value of $p$ in a differentially-rotating star. The toroidal field wound up by differential rotation will be:

$\frac{\partial B_{\phi}}{\partial t}=B_{z}^{\mathrm{init}} \varpi \frac{\partial \Omega}{\partial z}+B_{\varpi}^{\mathrm{init}} \varpi \frac{\partial \Omega}{\partial \varpi}$

where $B_{z}^{\text {init }}$ and $B_{\varpi}^{\text {init }}$ are the initial field components. We expect both spatial derivatives of $\Omega$ to tend to some finite value as $\varpi \rightarrow 0$, (i.e. near the $z$-axis,) whether the differential rotation is being driven by braking on the surface or by evolutionary changes in the moment of inertia, etc. There is no obvious reason why $B_{z}^{\text {init }}$ should go to zero on the axis, so the first term on the right-hand side of Eq. (5) will be proportional to $\varpi$. The behaviour of $B_{\varpi}^{\text {init }}$ near the $z$-axis is less certain, and it may have some dependence on $\varpi$ so that the second term goes as $\varpi^{2}$ or higher, but the two terms added together, and therefore the toroidal field component $B_{\phi}$, will be proportional to $\varpi$ as $\varpi \rightarrow 0$, i.e. $p=1$.

So if we use $p=1$, the only unstable mode is $m=1 ; m=0$ and $m=2$ are marginally stable. If $p=2$, then we should expect the $m=0, m=1$ and $m=2$ modes all to be unstable. 
Only high values of the vertical wavenumber $n$ are unstable. This is due to the dominant effect of the stable stratification. In order to minimise the energy lost against the stable buoyancy force, the vertical displacements have to be small compared to the horizontal displacement. Since the displacements also have to be nearly incompressive (otherwise energy is lost in doing work against the pressure force) this implies that the vertical length scale is small.

If we define a local Alfvén frequency

$\omega_{\mathrm{A}} \equiv v_{\mathrm{A}} / \varpi=B /(\varpi \sqrt{4 \pi \rho})$,

then we expect the growth rate for a non-rotating star to be (Tayler 1973 and Goossens et al. 1981)

$\sigma \sim \omega_{\mathrm{A}} \quad\left(\Omega \ll \omega_{\mathrm{A}}\right)$.

The instability condition is local (in a meridional plane): if it is satisfied at some point $(\varpi, z)$, an unstable eigenfunction can be fit into a small (for adiabatic instability: infinitesimal) region around this point (Tayler 1973). The instability is thus of an "interchange" type. This property holds by virtue of the assumption of a purely toroidal field. It greatly simplifies the analytical stability analysis, and allows a detailed treatment of the effects of diffusion and viscosity (Acheson 1978). In the azimuthal direction, the instability is global, since only low azimuthal orders $m$ are unstable. Connected with this is the fact that the typical instability time scale (if conditions for instability are satisfied) are always of the order of the time it takes an Alfvén wave to travel around the star in the azimuthal direction.

If the star is rotating with the rotation axis parallel to the magnetic axis, a stabilising effect is produced so that instead of Eq. (4) we have (Pitts \& Tayler 1986)

$p>\frac{m^{2}}{2}+1 \quad(m \neq 0) \quad$ and $\quad p>1 \quad(m=0)$

in the limiting case where the rotational velocity is much greater than the magnetic. In the particular case of $p=1$, we expect stability at all values of $m$. (We note that this is a peculiarity of the adiabatic case: when magnetic and thermal diffusion are included, the conditions on $p$ for instability reverts to Eq. (4) (cf. Appendix in Spruit 1999 and below).)

\subsection{The effect of magnetic diffusion}

As mentioned, there is a lower limit on the vertical wavenumber of an unstable mode, caused by the work which must be done against gravity to move gas in the vertical direction. There is also an upper limit to $n$, caused by diffusion: the unstable perturbations diffuse away at a rate of order $\eta n^{2}$, so an instability whose intrinsic (adiabatic) growth rate is less than its decay rate by diffusion will be smothered. The range of unstable radial wavenumbers for the $m=1$ mode is therefore, approximately, (Acheson 1978; Spruit 1999):

$\frac{\sigma}{\eta}>n^{2}>\frac{N^{2}}{\omega_{\mathrm{A}}^{2} r^{2}}$,

where $r$ is some measure of the size of the field in the horizontal direction. This is the condition in the absence of thermal diffusion $(\kappa=0)$. A complication arises here because it turns out that in the presence of both thermal and magnetic diffusion, the limit $\kappa / \eta \rightarrow 0$ is a singular one with respect to the instability condition: there exist double diffusive instabilities which are absent in the case $\kappa=0$. Since there is always some thermal diffusion due to numerical effects, we should not expect the simulations to reproduce (9) accurately. This is discussed further below.

For a $p=1$ field with $\boldsymbol{B}=\left(0, B_{0} \varpi / \varpi_{0}, 0\right)$ in the slowlyrotating case $\Omega \ll \omega_{\mathrm{A}}$ we have unstable wavenumbers $n$, and hence instability, if (using Eq. (7))

$\omega_{\mathrm{A}}^{3}>\frac{\eta N^{2}}{r^{2}}$

Instability is thus suppressed at low field strengths. The instability condition is a function of the meridional coordinates $(\varpi, z)$.

\subsection{The effect of thermal diffusion}

Thermal diffusion has the effect of reducing the stabilising effect of the density stratification, allowing instability for a larger range of vertical wavenumbers. This is a general effect, not only for the magnetic instabilities discussed here. It was first noted in the astrophysical context by Zahn (1974). It is important in stars since the thermal diffusion (measured by the diffusivity, with units area per unit time) is much faster than other damping effects (like magnetic and viscous diffusion). In this case there exist length scales large enough that these other damping effects are negligible on the time scale on which the instability operates, but at the same time small enough that thermal diffusion can wipe out the temperature fluctuations due to vertical displacement against the stable thermal stratification. This led to Zahn's (1974, 1983) formulation of shear instability in stellar interiors, widely used in stellar evolution calculations.

The effect can be incorporated by replacing the buoyancy frequency frequency $N$ by a value $\tilde{N}$ given by

$\tilde{N}^{2}=\frac{N^{2}}{1+\tau_{\mathrm{I}} / \tau_{\mathrm{T}}}$,

where $\tau_{\mathrm{I}}$ is the timescale of the instability, i.e. $1 / \sigma$, and $\tau_{\mathrm{T}}$ is the thermal timescale, equal to $1 / \kappa n^{2}$. [This is correct by order of magnitude for all instabilities, and can be made more exact for any given one.] This should manifest itself in a decrease of the minimum unstable wavenumber given in Eq. (9) above; it has exactly the same effect as a reduction in the acceleration of gravity $g$.

\subsection{The model}

To simulate conditions appropriate to a stratified stellar interior, without simulating the star as a whole, we make use of the fact that the Tayler instability is always present in at least some region near the magnetic axis. It has a low threshold, being suppressed only at very low field strengths where the combined effects of vertical stratification and magnetic diffusion suppress the instability (cf. Eq. (10)). We use a local plane-parallel approximation of the star, with the direction of gravity parallel to the magnetic axis. This is a good approximation for a small region near the axis at the low field strengths of primary interest, since the (meridional components of the) unstable wavenumbers are then high (cf. Eqs. (9) and (10)).

The ideal gas equation of state, measuring temperature in units such that the molar gas constant divided by the molecular mass is unity, is

$P=\rho T \quad$ and $\quad e=\frac{T}{\gamma-1}$.

The momentum equation:

$\frac{\mathrm{D} \boldsymbol{u}}{\mathrm{D} t}=-\frac{1}{\rho} \boldsymbol{\nabla} P+\frac{1}{\rho} \boldsymbol{J} \times \boldsymbol{B}+\boldsymbol{g}+2 \boldsymbol{u} \times \boldsymbol{\Omega}$. 
Conservation of mass:

$\frac{\mathrm{D} \rho}{\mathrm{D} t}=-\rho \nabla \cdot \boldsymbol{u}$.

Specific internal energy: (thermal conduction and Ohmic heating)

$$
\frac{\mathrm{D} e}{\mathrm{D} t}=-T \nabla \cdot \boldsymbol{u}+\gamma \kappa \frac{1}{\rho} \nabla \cdot(\rho \nabla e)+\eta \frac{1}{\rho} J^{2} .
$$

Electric current:

$\boldsymbol{J}=\nabla \times \boldsymbol{B}$,

and the induction equation:

$$
\frac{\partial \boldsymbol{B}}{\partial t}=-\nabla \times(\eta \boldsymbol{J}-\boldsymbol{u} \times \boldsymbol{B})
$$

where in the above equations, the velocity field is denoted by $\boldsymbol{u}$, the differential operator $\mathrm{D} / \mathrm{D} t \equiv \partial / \partial t+\boldsymbol{u} . \nabla, \eta$ and $\kappa$ are the magnetic and thermal diffusivities respectively, $e$ is specific internal energy and $\boldsymbol{g}$ is gravitational acceleration. We ignore fluid viscosity.

In the calculations, we have left out the ohmic heating term $\eta J^{2} / \rho$ from the energy Eq. (15). In a real star, there are other sources and sinks of energy (radiation) which together determine its thermal equilibrium structure. Since the magnetic energy $B^{2}$ is small, thermal changes due to Ohmic dissipation are slow compared with the (Alfvénic) time scale of interest here, it is more consistent to leave out the gradual heating by Ohmic diffusion together with these other sources of slow thermal change.

\section{3D MHD simulations}

\subsection{The numerical code}

We use a three-dimensional MHD code developed by Nordlund $\&$ Galsgaard (1995). The code uses a Cartesian coordinate system. This has the advantage, over a code using cylindrical coordinates, not only that the code is significantly simpler, but that it avoids the coordinate singularity on the axis, known to cause serious problems in essentially all grid-based methods in cylindrical or spherical coordinates. The disadvantage is that an intrinsically round peg (the toroidal field) has to be fitted into a square hole. The penalties are some waste of computing time (the grid points in the corners being unused), and a small amount of startup noise because a good initial equilibrium state is harder to construct. The boundary conditions are periodic; this is described in Sect. 3.4.

The code uses a staggered mesh, so that variables are defined at different points in the gridbox. For example, $\rho$ is defined in the centre of each box, but $u_{x}$ in the centre of the face perpendicular to the $x$-axis, so that the value of $x$ is lower by $\frac{1}{2} \mathrm{~d} x$. Interpolations and spatial derivatives are calculated to fifth and sixth order respectively. The third order predictor-corrector timestepping procedure of Hyman (1979) is used.

For numerical stability, the code contains high-order damping terms. These were however switched off in this study - we were interested in the early (and therefore linear) stage of instability growth, before displacements and velocities become large enough to necessitate any stabilisation.

\subsection{Definition of the stratification}

We want to investigate, amongst other things, which vertical wavenumbers are unstable. This is achieved most easily if the growth rates of the instabilities, and indeed all timescales, are independent of $z$. To this end $T$ has to be made independent of $z$, so that the sound crossing time over the width of the box is independent of height. The stratification is thus approximated by that of an isothermal atmosphere. This is sufficient for the present purpose, since it allows the inclusion of the essence of the stabilising effect of buoyancy.

The equilibrium magnetic field $\boldsymbol{B}$ is then made dependent on $z$ in such a way that the ratio of thermal and magnetic energy densities, $\beta=e \rho 8 \pi / B^{2}$, and therefore the Alfvén speed, is independent of $z$.

\subsection{Initial magnetic configuration and equilibrium}

Let $(\varpi, \phi)$ denote polar coordinates on a horizontal plane. We consider a purely azimuthal field of the form

$\boldsymbol{B}=B_{0}\left(\frac{\varpi}{\varpi_{0}}\right)^{p} \mathrm{e}^{-\varpi^{2} / \varpi_{0}^{2}} \mathrm{e}^{-z / 2 H} \boldsymbol{e}_{\phi}$,

where $\varpi_{0}$ is a length constant, set to a quarter of the horizontal size of the computational box, and $B_{0}$ is a constant which determines the strength of the magnetic field. The exponential containing $z$ keeps the value of $\beta$ independent of $z$ (see Sect. 3.4.2). The instability is in the centre, where $\varpi / \varpi_{0} \ll 1$. The exponential containing $\varpi$ is present to make the axisymmetric field fit inside a square computational box. As the field evolves during the instability, it will spread outwards so that some margin has to be left empty around the region containing the field. The results show that this has been successfully avoided, at least during the linear phase of growth.

To ensure that the initial state is close enough to equilibrium to convincingly measure the growth of the instability, the pressure, density and temperature fields have to be adjusted to the magnetic force brought about the field given in Eq. (18).

Let

$\rho(\varpi, z)=\rho_{0} W(\varpi) \mathrm{e}^{-g z / T_{0}} \quad$ and $\quad T(\varpi)=T_{0} D(\varpi)$.

The equilibrium conditions (Eqs. (12)-(17) with $\partial_{\mathrm{t}}=\boldsymbol{v}=\eta=0$ ) are then satisfied for the axisymmetric field given by Eq. (18) provided that

$$
\begin{aligned}
& W(\varpi)=1+\frac{B_{0}^{2}}{4 \rho_{0} T_{0}} \mathrm{e}^{-2 \varpi^{2} / \varpi_{0}^{2}} \quad \text { and } \\
& D(\varpi)=1-\frac{2 \varpi^{2} / \varpi_{0}^{2}}{1+\frac{4 \rho_{0} T_{0}}{B_{0}^{2}} \mathrm{e}^{2 \varpi^{2} / \varpi_{0}^{2}}}
\end{aligned}
$$

for the case $p=1$, or, if $p=2$,

$$
\begin{aligned}
& W(\varpi)=1+\frac{B_{0}^{2}}{8 \rho_{0} T_{0}}\left(1+2 \frac{\varpi^{2}}{\varpi_{0}^{2}}\right) \mathrm{e}^{-2 \varpi^{2} / \varpi_{0}^{2}} \quad \text { and } \\
& D(\varpi)=1-\frac{4 \varpi^{4} / \varpi_{0}^{4}}{1+2 \frac{\varpi^{2}}{\varpi_{0}^{2}}+\frac{8 \rho_{0} T_{0}}{B_{0}^{2}} \mathrm{e}^{2 \varpi^{2} / \varpi_{0}^{2}}}
\end{aligned}
$$

Magnetic diffusion will destroy the equilibrium even in the absence of dynamic instability. This should not be problematic as long as the timescale over which the instability becomes visible 
is shorter than the timescale over which the equilibrium is lost in this way. This condition is comfortably fulfilled in the simulations presented here, because the instability operates on a small length scale. Magnetic diffusivity of a value which is relevant for the instability has little effect on the much larger length scale of this initial field configuration.

\subsection{Boundary conditions}

Under the conditions prevailing in stellar interiors, the expected length scale of the instabilities is very small (at least in the radial direction). As in the case of hydrodynamic instabilities, therefore, different parts of the star are in effect disconnected from each other as far as the instability is concerned. The computational box can then be taken to cover only a small part of the star, as is also done in studies of magnetic instability in thin accretion discs (e.g. Hawley et al. 1995). This raises a problem with the boundary conditions, since the box boundaries are special locations in the computational volume that have no counterpart in the real star. With rigid boundaries, spurious phenomena such as boundary layers would affect the results. Such effects are minimised by using periodic boundary conditions: there are then no special locations in the computational box.

\subsubsection{Horizontal}

Periodic conditions in the $x$ and $y$ directions are implemented by copying unknown values of $P, T, \rho$ and $\boldsymbol{v}$ outside the box boundaries from their values at $x \pm L, y \pm L$ where $L$ is the width of the box. For the magnetic field, the sign of $\boldsymbol{B}$ is reversed in the copying process, so that there are no current sheets at the boundaries. In any case, with the field used, the values at the boundaries are very small compared to the values at the centre of the box.

\subsubsection{Vertical}

In the vertical direction periodic conditions are also possible by making use of an invariance of the equations for the case of an isothermal stratification. By this symmetry, a shift in height is equivalent to multiplication of the variables by constant factors. In this way the variables can be scaled from the bottom to the top of the computational box. Thus, when interpolating or differentiating across the top and bottom of the computational box, we multiply or divide the density by a factor $\exp \left(L_{z} / H\right.$ ) (where $L_{z}$ is the height of the box and $H$ is the scale height). These "scaled periodic" boundary conditions are the appropriate equivalent of periodic conditions for a stratified medium.

Unfortunately, it turns out that this procedure does not work in its simplest form. Small perturbations launched by the initial disequilibrium propagate vertically, as a mixture of sound waves and internal gravity waves. The upward propagating components of a sound wave will grow (in the limit where the wavelength is much smaller than the scale height) at a rate of the order of the buoyancy frequency (differing from it by a factor 1.02 if $\gamma=$ $5 / 3$ ). If the wave leaving the top of the box is fed back in at the bottom, this amplification goes on indefinitely.

Though the initial perturbations can be made small, the exponential growth of the upward-travelling waves causes a problem if this growth is faster than that of the magnetic instability under study. Since we want to operate in the regime where $N \gg \omega_{\mathrm{A}}$, this will indeed be the case. Engineering fixes like artificial damping terms designed to affect sound waves would not greatly help, since internal gravity waves will also grow in this way at a comparable rate.

Many methods were tried to extinguish these waves. The most satisfactory solution found was to divide the volume vertically into two halves, with gravity pointing downwards and upwards in the top and bottom halves of the box respectively. A sound wave is still amplified within one half, but will not see any net increase in amplitude over the course of a journey through the whole box. The physics of the magnetic instability is replicated in the two halves, so that the penalty is a factor of two in computational cost.

\subsection{Initial perturbations}

All of the runs were started with a small perturbation to the velocity field. This could either be given at all values of $m$ and $n$, or just to one particular mode. There was some uncertainty as to the precise form of the perturbation required; how it should vary as a function of $\varpi$, whether it should include a vertical component and in what sense the phases of the two horizontal components should vary with $z$ were all unanswered questions. Several different formulations were tried, producing, reassuringly, virtually identical results. For the runs appearing here, the initial perturbation had its maximum at $\varpi=0$ and died away at increasing $\varpi$ so that at $\varpi=\varpi_{0}$ it was almost zero, the vertical component of the velocity perturbation was zero and for the $m \neq 0$ modes, the phase in the $\phi$ direction went from 0 to $2 \pi$ over the course of one vertical wavelength so that the cross-section remained the same at all $z$ but was rotated about the axis once per wavelength. The initial perturbation is therefore of the form:

$$
\begin{aligned}
\boldsymbol{v}= & \sum_{m=0, n} V_{0}^{n} \frac{\varpi}{\varpi_{0}} \exp \left(-\frac{5}{2} \frac{\varpi^{2}}{\varpi_{0}^{2}}\right) \cos (n z) \boldsymbol{e}_{\varpi} \\
& +\sum_{m \neq 0, n} V_{m}^{n} \exp \left(-3 \frac{\varpi}{\varpi_{0}}-\frac{\varpi^{2}}{\varpi_{0}^{2}}\right)\left[\cos (n z-m \phi) \boldsymbol{e}_{\varpi}\right. \\
& \left.+\sin (n z-m \phi) \boldsymbol{e}_{\phi}\right]
\end{aligned}
$$

The amplitude of the initial velocity perturbation $V_{m}^{n}$ was given a value $3 \times 10^{-5}$, which corresponds to a fraction $2 \times 10^{-5}$ of the sound speed or $2 \times 10^{-4}$ of the Alfvén speed. This value is large enough that the numerical perturbation is small in comparison, while being small enough to follow the linear phase of instability growth for several growth time-scales.

\subsection{Accessible parameter values}

The separation of time scales $\omega_{\mathrm{A}} \ll N$ and $\Omega \ll N$ appropriate for stars is also convenient for the analytical stability analysis. Such widely differing time scales are problematic for numerical simulations, however, since the time step will be set by the shortest time scale, the sound crossing time (which is of the order of $1 / N)$, while the phenomena of interest happen on the slower magnetic time scale. In the first set of calculations reported below we have set $\Omega=0$, and the ratio $\omega_{\mathrm{A}} / N$ is of order $0.01-0.1$. For such values, the predicted growth rates are already close to their asymptotic $\left(\omega_{\mathrm{A}} \ll N\right)$ value, so not much would be gained from more expensive calculations with lower field strengths. The effect of rotation is investigated in Sect. 4.4.

\section{Results}

To test the analytic predictions and the behaviour of the code a series of test cases was studied, starting with a simple 


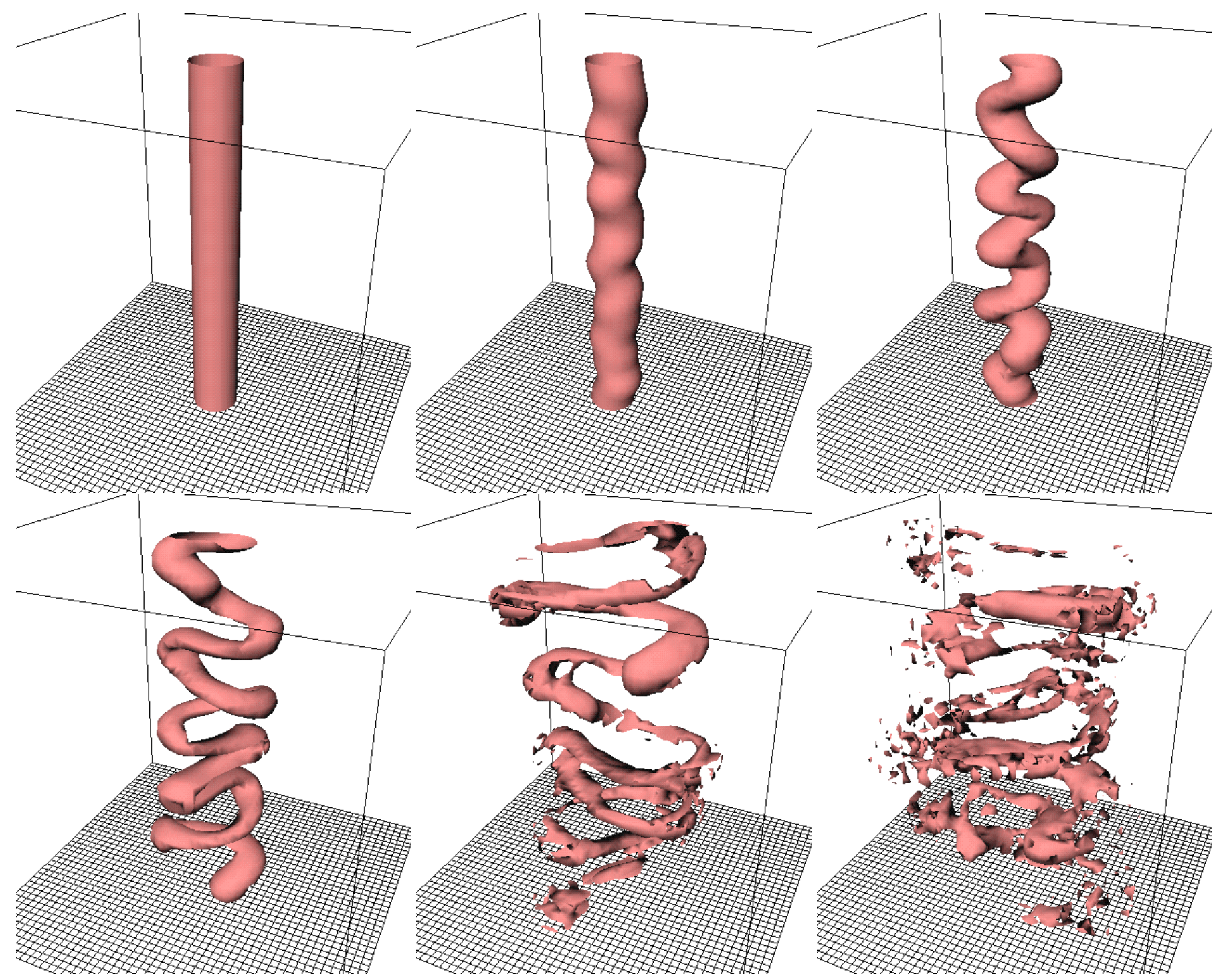

Fig. 2. Following the evolution of instabilities - surfaces of constant initial $\varpi=\pi / 10=\varpi_{0} / 5$ at roughly regular time intervals: $t=$ $6.30,9.07,10.86,12.52,14.56,16.20$.

unstratified medium with adiabatic conditions. Physical ingredients are then added concluding with the case of most interest for a stellar interior, in which stratification, magnetic and thermal diffusion are included.

\subsection{Dependence of stability on $p$}

Two initial field configurations, $p=1$ and $p=2$, were tried. A resolution of $48 \times 48 \times 48$ was used for these runs, and the computational box is a cube of side $2 \pi$. A zero value was used for $g$, and $B_{0}=1.28$. As in all runs $\gamma=5 / 3$ and $\varpi_{0}=\pi / 2$. This produces a maximum value of the magnetic energy density of one tenth of the thermal energy density, (i.e. $\beta=10$ ). This is still in the weak-field approximation $\left(v_{\mathrm{A}} \ll c_{\mathrm{s}}\right)$.

To follow the development of the instability in time, it was found useful to calculate the Lagrangian displacement field. This was computed by adding a vectorial tracer field $\chi=\left(\chi_{x}, \chi_{y}\right.$, $\left.\chi_{z}\right)$ to the code. The initial values of this field were simply the coordinates $x_{0}, y_{0}$ and $z_{0}$ at $t=0$, and it was evolved according to the equation $\mathrm{D} \chi / \mathrm{D} t=0$, making use of the same advection routines as in the rest of the code. The displacement field $(\xi)$ can be found by subtracting the tracer field from its initial value. This
Lagrangian displacement field was used for the representations shown in Fig. 2.

In Fig. 2 we can see the evolution of the displacement field near the magnetic axis: it shows how a fluid volume that was initially a cylinder around the axis evolves in time under the influence of the instability of the magnetic field, in the $p=1$ case. The surfaces shown are surfaces of constant $\chi_{x}^{2}+\chi_{y}^{2}$. Some magnetic diffusion $\left(\eta=3 \times 10^{-3}\right)$ has been added to remove the higher spatial frequencies and give the picture a more pleasing look - in the absence of diffusion, all spatial frequencies up to the Nyquist frequency (wavelength of two grid spacings) are present and it would be difficult to see the form of the instability. Is it clear that we are looking at an $m=1$ instability since the plasma is displaced in the horizontal direction without losing its circular cross-section.

In Fig. 3 we see the $p=1$ and $p=2$ runs compared; three surfaces of constant $\chi_{x}^{2}+\chi_{y}^{2}$ have been plotted, two of which have been partly cut away. These confirm that in the $p=1$ case, the instability grows most quickly on the axis, and that in the $p=2$ case, it grows most quickly away from the axis. This should be no surprise as here the local Alfvén frequency $\omega_{\mathrm{A}}$ (see Eq. (6)) is greatest. 

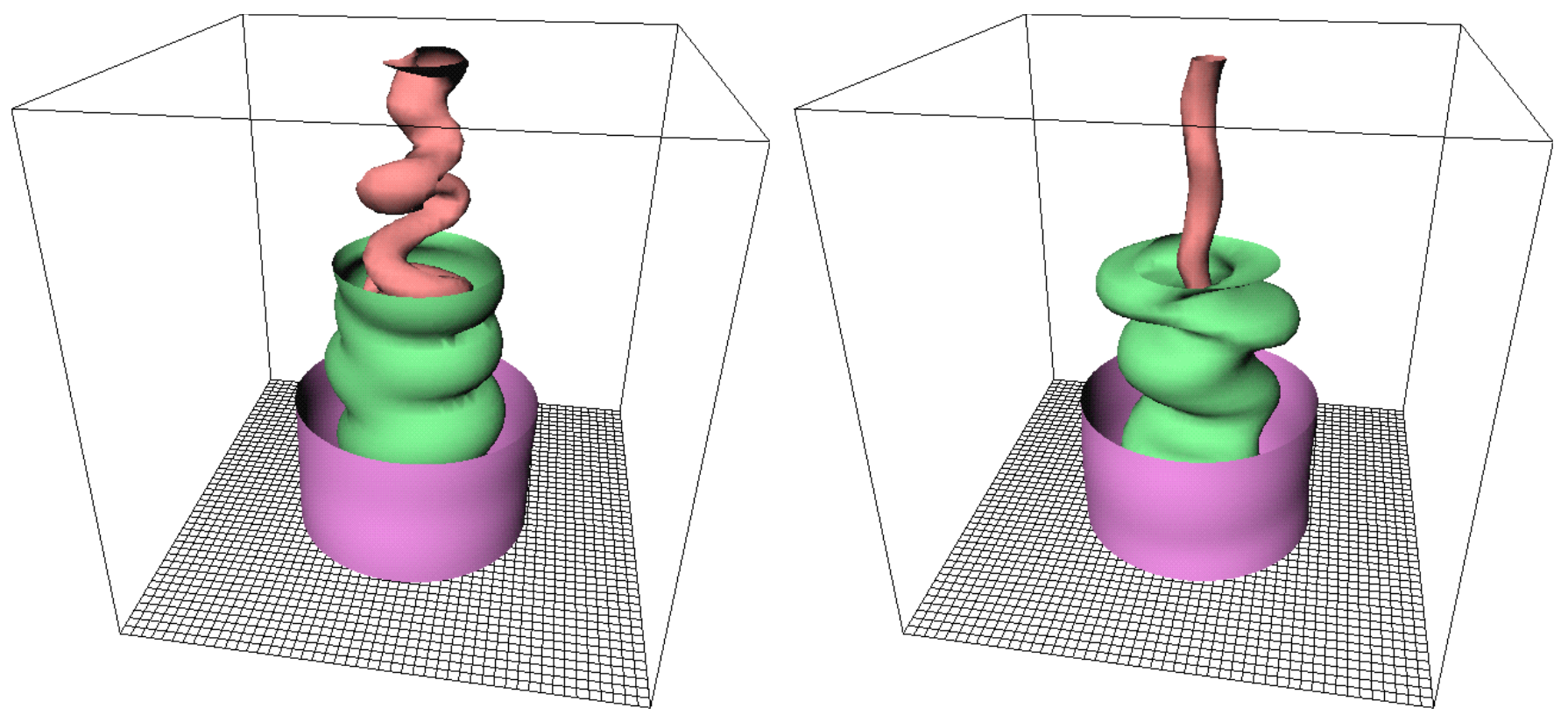

Fig. 3. Two runs, identical except for the value of $p$, which is equal to 1 and 2 in the left and right hand figures respectively. The left hand figure is the same as the fourth frame of the sequence in Fig. 2, i.e. at $t=12.52$. In the right hand figure, $t=15.29$. Plotted are three surfaces of constant initial $\varpi$ (i.e. constant $\chi_{x}^{2}+\chi_{y}^{2}$ ) inside one another. It is clearly visible that the instability is on the axis in the case of $p=1$, but away from the axis when $p=2$.

It is possible to perform a Fourier decomposition of the displacements in the $\phi$ dimension, enabling us to see the amplitudes of the different $m$ modes separately. We can write the displacement $\xi$ as

$\xi(\varpi, \phi, z, t)=\frac{1}{2} A_{0}(\varpi, z, t)+\sum_{m=1}^{\infty} \mathfrak{R}\left(\boldsymbol{A}_{m}(\varpi, z, t) \mathrm{e}^{\mathrm{i} m \phi}\right)$

and calculating

$\operatorname{Amp}_{m}(t)=\left(\frac{\int_{\varpi=0}^{\varpi_{\max }} \int_{z=0}^{L_{z}} \boldsymbol{A}_{m}^{*} \cdot \boldsymbol{A}_{m} 2 \pi \varpi \mathrm{d} \varpi \mathrm{d} z}{\pi \varpi_{\max }^{2} L_{z}}\right)^{\frac{1}{2}}$

should give us a suitable measure of the amplitudes of each $m$ mode. A value of $\varpi_{\max }=0.5 \varpi_{0}$ is used, since this is the region of interest, where the field strength is roughly proportional to $\varpi^{p}$. In any case, choosing a different value doesn't have any significant affect on the results. This quantity $\mathrm{Amp}_{m}$ (or rather, its logarithm) is plotted in Fig. 4 for the aforementioned $p=1$ run. We can see that the $m=1$ mode is the only unstable one when $p=1$.

It is possible to do this Fourier decomposition on not just the displacement field but also on the velocity field and on the (change in the) magnetic field, giving analogous amplitudes $\mathrm{Amp}_{m}^{\prime}$ and $\mathrm{Amp}_{m}^{\prime \prime}$ respectively. These quantities are plotted in Figs. 5 and 6. Whereas the amplitudes in the displacement field begin at zero, the amplitudes in the velocity field begin with a finite value, since we are giving the plasma an initial perturbation to the velocity field. The graph of amplitudes in the $\boldsymbol{B}-\boldsymbol{B}(t=0)$ field looks much like that of the displacement field. This is what we should expect, given that the magnetic surfaces and fluid surfaces should tend to follow each other, at least in the limit of low diffusion.

At some point the other modes begin to grow also, presumably when the $m=1$ mode enters the non-linear phase. To test this hypothesis, we can consider that the smallest vertical wavelength present is two grid boxes, equal to a distance 0.26 , so the minimum value of $1 / n$ will be $\lambda_{\min } / 2 \pi=0.042$. The nonlinear phase begins once the displacements become comparable to this. Figure 7 shows the maximum value in the displacement field, i.e. the maximum displacement from equilibrium reached anywhere in the computational box - we can confirm that this value reaches $\lambda_{\min } / 2 \pi$ at roughly the same time as the $m \neq 1$ modes begin to grow.

In the case of $p=2$, the instability grows more quickly away from the axis. We have already seen this in Fig. 3. This complicates the analysis, as the instability is strongest in the region where the initial field deviates from proportionality to $\varpi^{p}$. This is to be expected, since the growth rate is of order $v_{\mathrm{A}} / \varpi$ which is proportional to $\varpi$ if $p=2$. However, it should be possible to use data from the inner region only, before it is contaminated by non-linear development from the outer region. Figure 8 is the equivalent of Fig. 4 for the $p=2$ case. We can see that the $m=0$ and $m=2$ modes are growing, as well as the $m=1$ as in the previous case.

All subsequent discussion is limited to the physically more likely $p=1$ case.

\subsection{Stratified and non-stratified runs and vertical wavenumber}

For the runs described above, we were after just a qualitative result, as opposed to the quantitative result required from the following runs, which had consequently to be set up in a slightly different way.

In some cases we were interested in the behaviour at different vertical wavelengths. We therefore performed a second Fourier decomposition of the displacement (or velocity) field, this time in the vertical direction. This yields the amplitude at each value of $m$ and $n$ (values of the latter correspond to wavelengths from the height of the box down to two grid spacings):

$\boldsymbol{A}_{m}(\varpi, z, t)=\frac{1}{2} \boldsymbol{C}_{m 0}(\varpi, t)+\sum_{n} \mathfrak{R}\left(\boldsymbol{C}_{m n}(\varpi, t) \mathrm{e}^{\mathrm{i} n z}\right)$. 


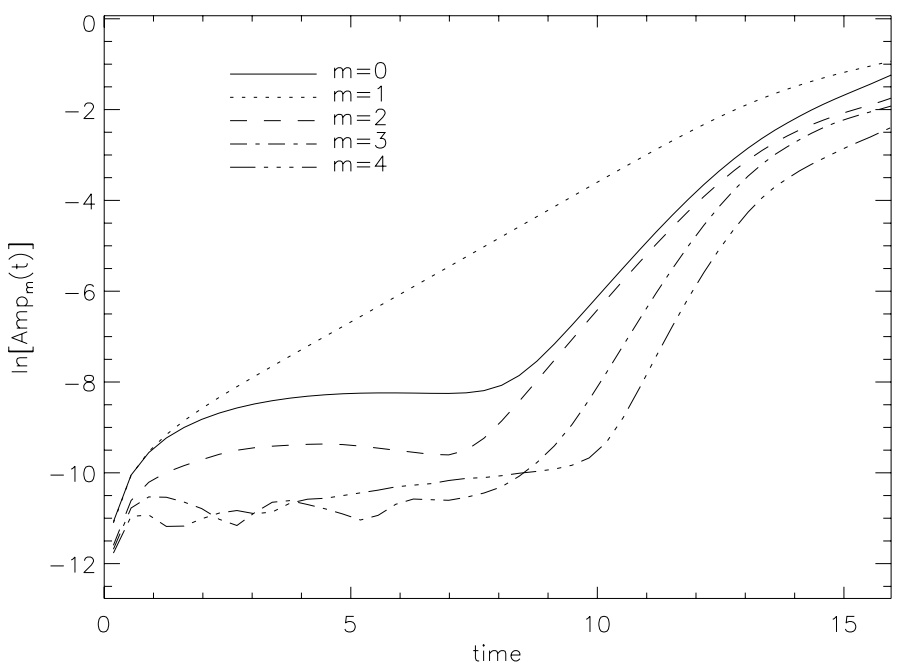

Fig. 4. Displacement amplitudes of different $m$ modes, when $p=1$. Adiabatic $(\kappa=\eta=0)$ unstratified case.

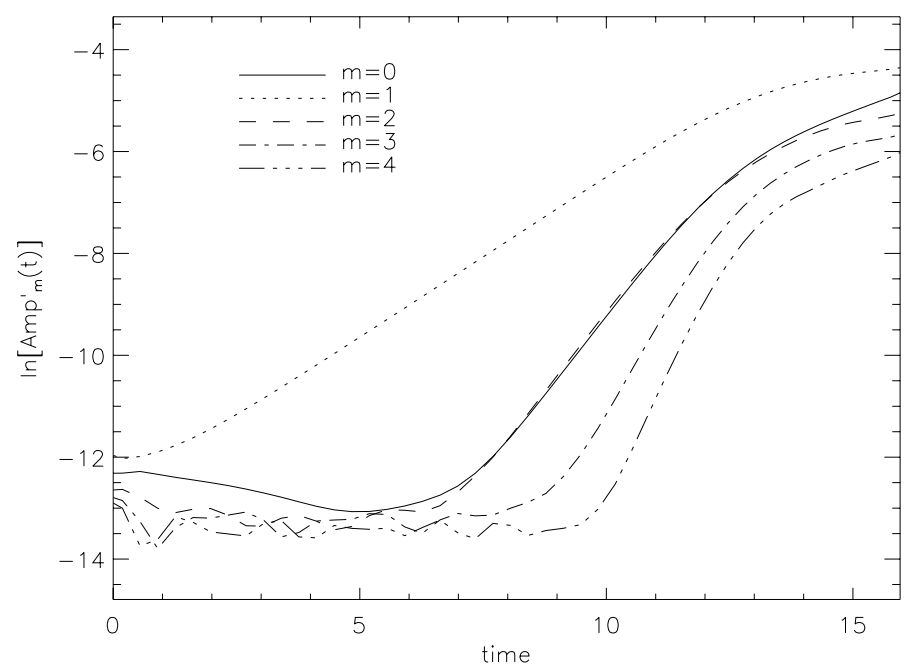

Fig. 5. Velocity amplitudes of different $m$ modes, when $p=1$. Adiabatic unstratified case.

As before (see Eq. (26)), an integration of the coefficients $\boldsymbol{C}_{m n}$ over $\varpi$ can be done out to a value $\varpi_{\max }$, and it is this amplitude $\operatorname{Amp}_{m n}(t)$ which is used for the following analysis.

In finding the dependence of instability growth at different vertical wavelengths as a function of some parameter, (e.g. magnetic diffusion), one has two options: to measure the growth at several wavelengths in a single run with some certain value of the parameter in question, or to measure the growth at a single wavelength in several runs, each with a different value of the parameter. Two computational restrictions made the second method the most practical: firstly, it was discovered that at wavelengths comparable or greater than $\varpi_{0}$ grew more slowly than expected - an effect purely of the dimensions of the computation box, this meant that the maximum wavelength which could be examined was $\pi / 10$, i.e. one fifth of $\varpi_{0}$; secondly, the code could not resolve very short wavelengths - the minimum wavelength we could look at reliably was eight grid spacings.

In the cases where stratification was not necessary, therefore, a vertical resolution of 8 was sufficient, with a box of dimensions $2 \pi \times \pi / 10$; a horizontal resolution of 72 proved more than enough. In cases where it was necessary to hold to the $N \gg \omega_{\mathrm{A}}$ condition, we still looked at a wavelength of $\pi / 10$

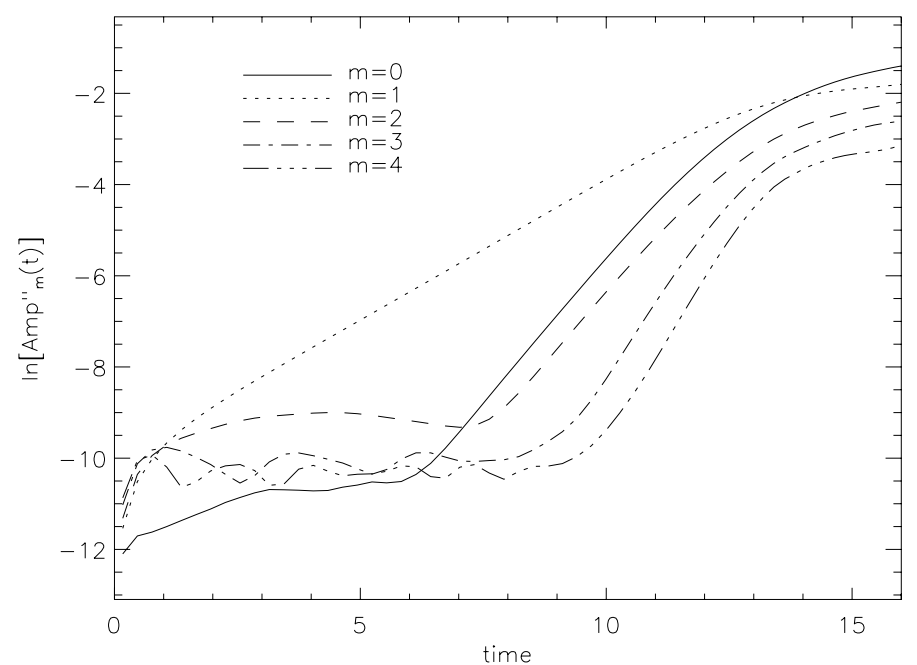

Fig. 6. $B$-field amplitudes of different $m$ modes, when $p=1$. Adiabatic unstratified case.

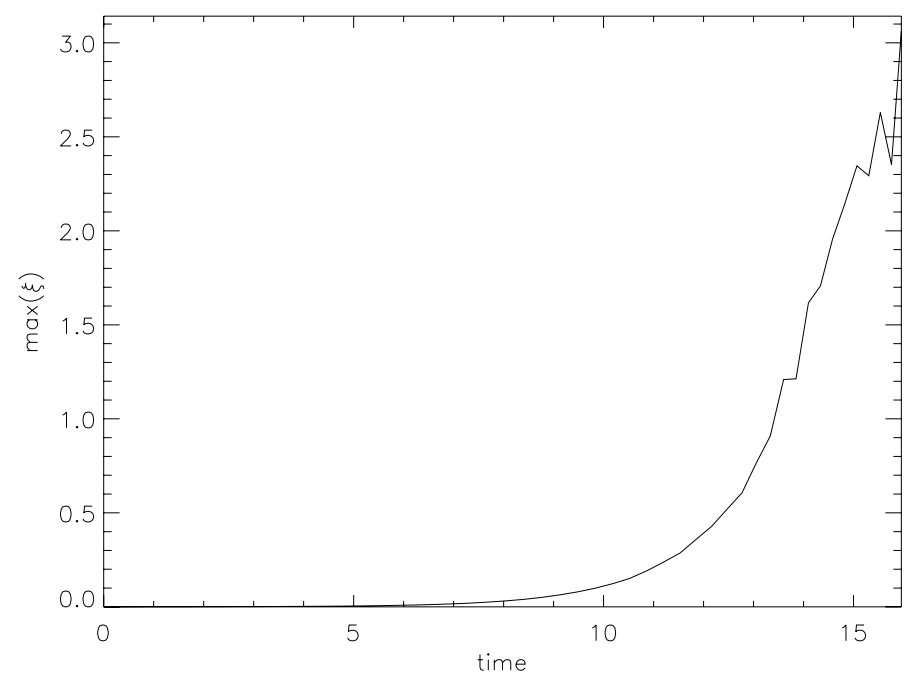

Fig. 7. Maximum displacement, when $p=1$. The computational box is a cube of side $2 \pi$, and the shortest wavelengths of the instability may be just a twenty-fourth of this distance. Adiabatic unstratified case.

(corresponding to a wavenumber of 20), but implemented the scheme described in Sect. 3.4.2, so that the gravity was in opposite directions in the two halves of the box. A vertical resolution of 64 was then used in a box of size $2 \pi \times 4 \pi / 5$. All of the following runs were done with one of these two setups. Since stratification has a wavelength-dependent effect, i.e. the stratification has a greater effect on longer wavelengths (see Eq. (9)), phenomena which either do not depend on wavelength or only effect the short wavelengths can be investigated using the non-stratified model with zero gravity. The first three of the following sections fall into this category: field strength, rotation and magnetic diffusion. As a check, the runs with rotation were also done with stratification. The stratified setup is however required to look, in the last two sections, at the effect of gravity (obviously) and of thermal diffusion.

\subsection{Dependence of growth rate on field strength}

The growth rate is expected to be proportional to the field strength. To see whether this is the case, five runs were done with $B_{0}$ equal to $1.28,0.404,0.128,0.0404$ and 0.0128 respectively. 


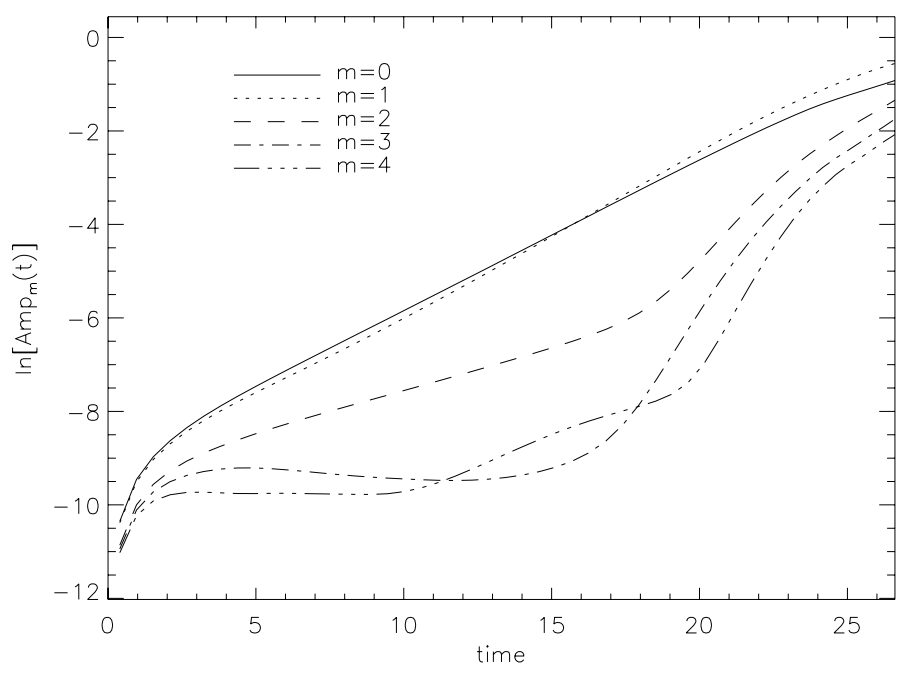

Fig. 8. Displacement-field amplitudes of different $m$ modes, when $p=$ 2. Adiabatic unstratified case.

These correspond to minimum values (that is, where the field is strongest) of $\beta$ of 10 to $10^{5}$. Magnetic and thermal diffusivity were set to zero, as was gravity $g$ - as described in Sect. 4.2 for this non-stratified case, a resolution of $72 \times 72 \times 8$ was used.

The expected growth rate is given by (from Eqs. (6) and (7)):

$\sigma \sim \omega_{\mathrm{A}}=\frac{B_{0}}{\varpi_{0} \sqrt{4 \pi \rho}}$.

Figure 9 shows the maximum growth rate reached in the velocity field of this $m=1, n=20$ mode in the five runs, against the value of $\omega_{\mathrm{A}}$. The velocity field was more convenient for this purpose than the displacement field, as the growth rate in the latter begins at infinity (because the perturbation is to the velocity field) and falls to a steady value, before falling again once the non-linear stage is reached; the growth rate in the velocity field begins at zero and rises to a steady value before falling again - its maximum value is therefore reached during the period of steady growth. It can be seen from Fig. 9 that the growth rate $\sigma$ is proportional to the Alfvén frequency $\omega_{\mathrm{A}}$, and is in fact almost equal to it.

The growth rates have been predicted using the approximation that the magnetic energy density is very much less than the thermal, and that the Alfvén speed is very much less than the sound speed. In the run with the highest field strength these ratios are only 10 and 2.4 , which may explain the slightly lowerthan-expected growth rate here.

At this point, we can have a look at the growth of the poloidal field component, since the production of a poloidal component from toroidal component via this instability is part of the motivation for this study. We can decompose the field into its three components (in cylindrical coordinates). The strong-field run (with $\left.B_{0}=1.28, \omega_{\mathrm{A}}=0.81\right)$ is used for this purpose, and the (log of the) energy contained in the three components is plotted in Fig. 10, i.e. a volume integrations of $B_{\phi}^{2} / 8 \pi, B_{\varpi}^{2} / 8 \pi$ and $B_{\mathrm{z}}^{2} / 8 \pi$ at $\varpi<\varpi_{\max }=\varpi_{0} / 2$, as in Eq. (26). It can be seen in the graph that the energy in the poloidal components $\left(B_{\varpi}\right.$ and $\left.B_{\mathrm{z}}\right)$ is growing exponentially, with a growth rate double that of the growth in the displacement field - owing to the $B^{2}$ dependence.

In Fig. 11 is plotted the (log of the) energy in the $B_{z}$ component against the Alfvén time, i.e. $t / \omega_{\mathrm{A}}^{-1}$, for the five runs of different field strength $B_{0}$ described above. It can be seen that the growth rate of the poloidal field is indeed proportional to $\omega_{\mathrm{A}}$, just

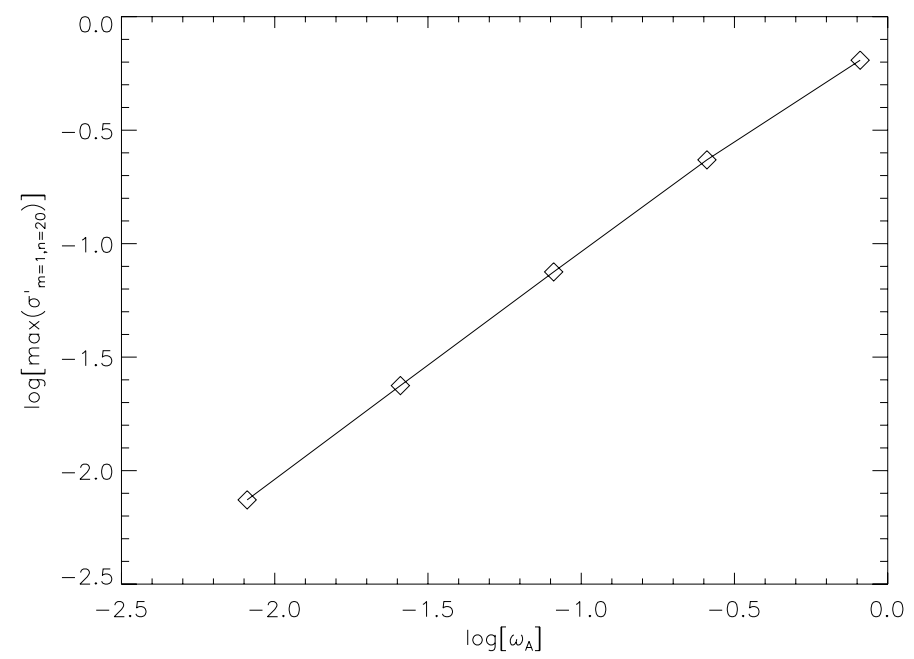

Fig. 9. The growth rate of the $m=1$ mode is roughly equal to $\omega_{\mathrm{A}}$, which is proportional to field strength. Adiabatic unstratified case.

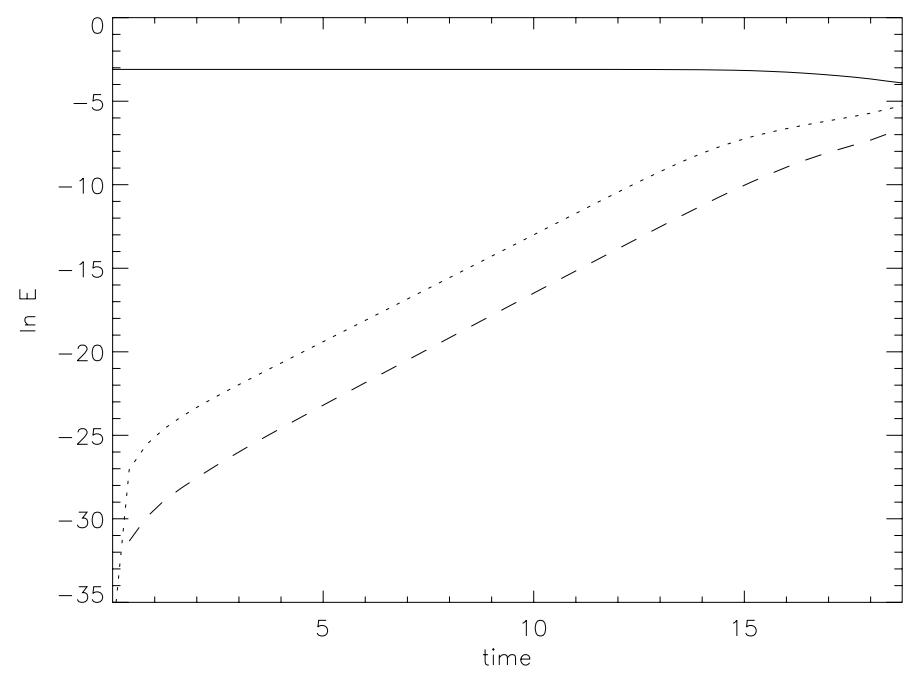

Fig. 10. The energy in the three components of the magnetic field. The solid line represents $B_{\phi}$, dotted $B_{\varpi}$ and dashed $B_{z}$. The two poloidal components are growing exponentially on a time-scale comparable to the Alfvén crossing time. Adiabatic unstratified case.

as the growth rate in the displacement field in Fig. 9. Note that the growth rate in the strongest-field run is again a little lower than expected, for the reason described above.

\subsection{Rotation}

We shall now look at the effect of rotation on the instability. An angular velocity is added (by means of a Coriolis force), parallel to the magnetic axis and gravity. In the case of $p=1$ we expect, looking at (8), to see the instability suppressed if $\Omega \gg \omega_{\mathrm{A}}$. A number of non-stratified $(g=0)$ runs were executed, using, as before, a resolution of $72 \times 72 \times 8$, with $\eta=\kappa=0$. The values 0 , $0.15,0.3,0.45,0.6,0.65,0.7,0.75,0.9$ and 1.2 of $\Omega$ were used, at a value of $B_{0}$ of 1.28 , which gives a growth rate in the absence of rotation of 0.64. In Fig. 12 the amplitude of the $m=1$ mode (as taken from the displacement field) is plotted for each of the runs, as a function of time. It can be seen that the instability is indeed suppressed if $\Omega$ is above a certain value somewhere between 0.65 and 0.70 . Above this value a distinct oscillatory behaviour sets in, indicating stability. 


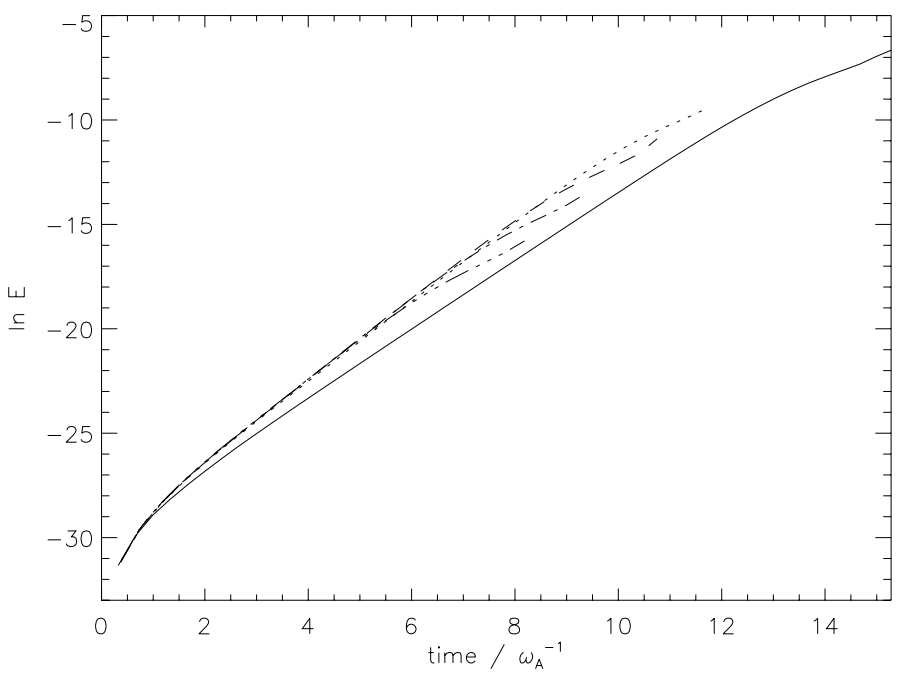

Fig. 11. The energy of the vertical component of the magnetic field $\left(B_{\mathrm{z}}^{2} / 8 \pi\right)$ against Alfvén time, for five runs with different magnetic field strengths. The solid, dotted, dashed, dot-dashed and dot-dot-dot-dashed lines represent runs with $B_{0}=1.28,0.404,0.128,0.0404,0.0128$. The growth rate of the energy in the vertical component of the magnetic field is roughly is roughly equal to $2 \omega_{\mathrm{A}}$. Adiabatic unstratified case.

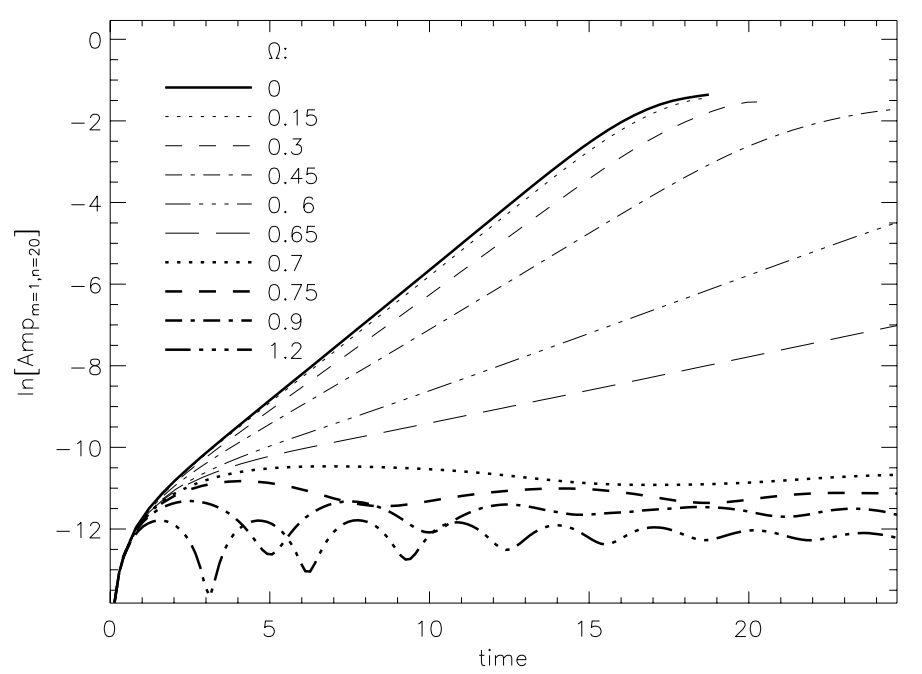

Fig. 12. Amplitudes of the $m=1, n=20$ mode at different values of $\Omega$, taken from the displacement field. Adiabatic unstratified case. For values of $\Omega$ of 0.7 and higher, an oscillatory behaviour is seen, indicating stability.

In order to check that this result holds in the regime $N \gg \omega_{\mathrm{A}}$, the runs were repeated using the stratified setup (see Sect. 4.2) and $g=9.1$, giving $N=5.8$ so that both $N \gg \omega_{\mathrm{A}}$ and $N \gg \Omega$ were fulfilled. This was found to make no difference at all to the suppression of the instability, there still existing a critical value of $\Omega$ roughly equal to the non-rotating growth rate above which the instability was suppressed.

\subsection{Magnetic diffusion and its effect on high wavenumbers}

We expect to to see an upper limit on $n$ due to magnetic diffusion, which is the same as saying that we expect to see a maximum value of $\eta$ at which the instability is seen, at a given value of $n$. As explained in Sect. 4.2, we have to look either at the growth of several $n$ modes at a given value of diffusion $\eta$, or to look at the growth of a particular $n$ mode in runs with different values

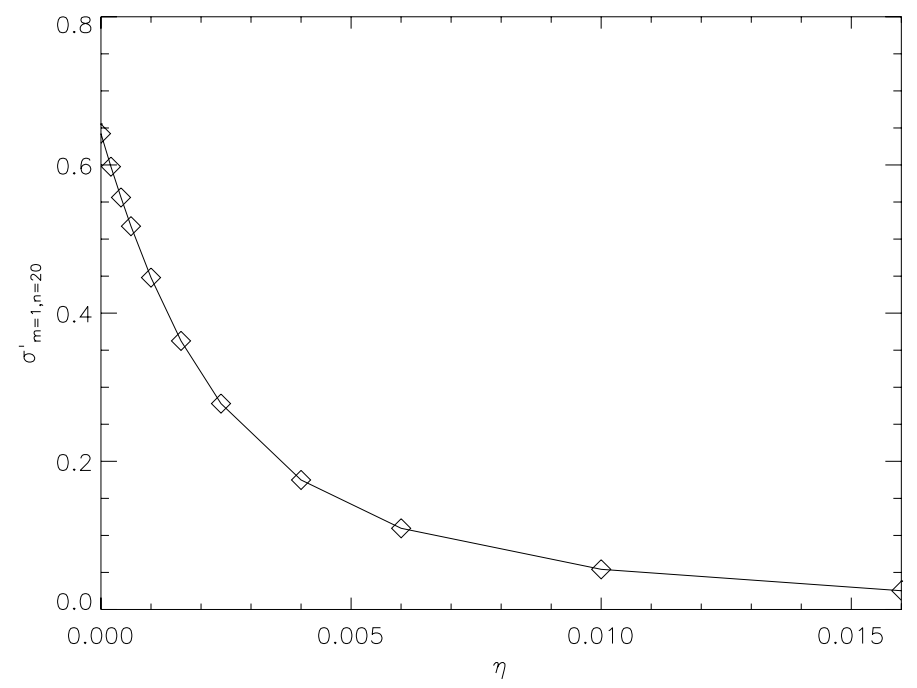

Fig. 13. Growth rate of the $m=1, n=20$ mode in the velocity field as function of the magnetic diffusivity $\eta$. Unstratified case.

of $\eta$. It was found that the latter was easier to perform, hence we expect to see, for a given value of $n$, a value of $\eta$ above which no instability is seen. For these runs, the non-stratified setup was used as gravity has no effect on this high-wavenumber limit.

We used $B_{0}=1.28$ as in the previous section, and the following values of magnetic diffusivity $\eta$ : $0,2 \times 10^{-4}, 4 \times 10^{-4}, 6 \times$ $10^{-4}, 10^{-3}, 1.6 \times 10^{-3}, 2.4 \times 10^{-3}, 4 \times 10^{-3}, 6 \times 10^{-3}, 10^{-2}$ and $1.6 \times 10^{-2}$.

Measuring the growth of the mode at wavenumber $n=20$ as a function of $\eta$, we expected to find a value of diffusion $\eta_{\text {crit }} \sim \sigma_{0} / n^{2}=1.6 \times 10^{-3}$ (from Eq. (9)) above which the instability does not grow, where $\sigma_{0}$ is the growth rate in the absence of diffusion. Figure 13 shows the growth rate of the velocity field measured at the time at which the growth rate in the zero diffusion (adiabatic) case is at its maximum $(t=10.4)$. It can be seen in the figure that the instability is not entirely suppressed at $\eta=\eta_{\text {crit }}$, just slowed by a factor of two or so. Even when $\eta$ is much higher than $\eta_{\text {crit }}$ the growth rate is still above zero. The reason for this is unclear - this is discussed in Sect. 5.

\subsection{Gravity and its effect on low wavenumbers}

We also expect to see a lower limit on the unstable vertical wavelength $n$ determined by the value of $g ; n_{\min }=N / \omega_{\mathrm{A}} r$ (Eq. (9)).

For these runs we obviously have to use the stratified setup, the growth of the same mode, $n=20$, being measured as in the previous three sections. All of the runs had $B_{0}=0.102$ and $\eta=\kappa=0$, each run then having a different value of gravitational acceleration $g$; the expected value of $g$ above which the $n=20$ mode does not grow is $g_{\text {crit }}=n B_{0} \sqrt{5 T / 8 \pi \rho}=10 / \pi$; the code was run with the following multiples of this value: $0,0.6,0.8,1,1.2,1.4$ and 2. Figure 14 shows the amplitude of the $m=1, n=20$ mode (calculated from the velocity field) for these values of $g$. As the figure shows, the instability stops growing at the expected value $g=g_{\text {crit }}$, within the measurement accuracy.

\subsection{Thermal diffusion}

If thermal diffusion is added, we expect to see a decrease in the minimum unstable vertical wavenumber, since the stable thermal buoyancy is reduced by diffusion on small length scales. To investigate this, thermal diffusion is added to the run in the 


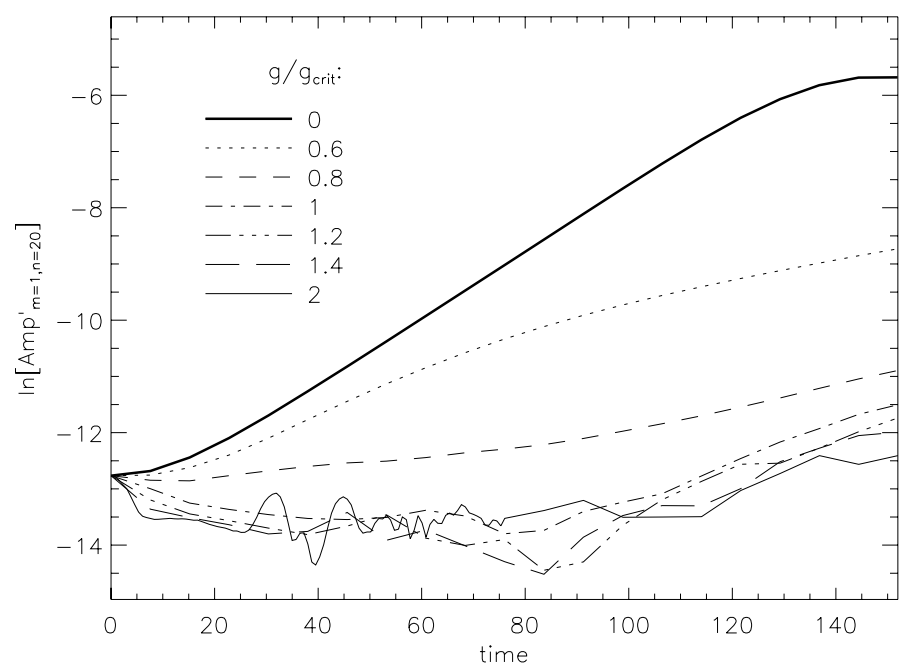

Fig. 14. Effect of the stabilising stratification, under adiabatic conditions $(\kappa=\eta=0)$. Velocity field amplitudes of the $m=1, n=20$ mode as a function of time for seven values of the acceleration of gravity $g$. The first half of the run with $g=2 g_{\text {crit }}$ is plotted at higher time resolution.

previous section where $g=g_{\text {crit }}=10 / \pi$, i.e. the borderline case where gravity is just strong enough to suppress the instability. The values of $\kappa$ used were $3 \times 10^{-5}, 10^{-4}, 3 \times 10^{-4}, 10^{-3}$, $3 \times 10^{-3}$ and $10^{-2}$. The results can be seen in Fig. 15. The figure also shows, for comparison, the case where $g=0$ and $\kappa=0$.

From Eqs. (9) and (11), we have

$n_{\min }^{2}=\frac{N^{2}}{\omega_{\mathrm{A}}^{2} r^{2}\left(1+\tau_{\mathrm{I}} / \tau_{\mathrm{T}}\right)} \propto \frac{g^{2}}{1+\kappa n_{\min }^{2} / \sigma}$.

We expect, therefore, that an increase in both $g$ and $\kappa$ can cancel each other out. It would be reasonable to expect that if we increase $g$ from $0.8 g_{\text {crit }}$ to $g_{\text {crit }}$, we need to increase $\kappa$ from zero to $\left((1.0 / 0.8)^{2}-1\right) \sigma / n^{2}$ to cancel out the effect on the instability. This works out as $\kappa=9 \times 10^{-5}$. Likewise, from $g=0.6 g_{\text {crit }}$ we need $\kappa=2.7 \times 10^{-4}$. This means that in runs with $g=g_{\text {crit }}$, using $\kappa=9 \times 10^{-5}$ and $2.7 \times 10^{-4}$ should produce the same instability growth rate in the $m=1, n=20$ mode as if we had reduced $g$ to $0.8 g_{\text {crit }}$ and $0.6 g_{\text {crit }}$ respectively, keeping $\kappa=0$. As Fig. 15 shows, these numbers are roughly in agreement with the numerical results.

\section{Discussion}

We set out in this study to verify, with numerical methods, the results of the existing analytical work on the instabilities of toroidal fields in stars, and to check that these results were relevant, e.g. that a predicted instability is not drowned out by stronger unstable modes that might have been missed, for example due to simplifying assumptions. The results have been largely positive.

Previous analytic work makes predictions of the dependence of instability conditions and growth rates on the parameters of an azimuthal magnetic field. These are the dependence of the field strength on distance $\varpi$ from the axis (the index $p=\mathrm{d} \ln B / \mathrm{d} \ln \varpi$ ), the stability of the stratification (measured by the buoyancy frequency $N$, or equivalently the acceleration of gravity $g$ ), the rotation rate $\Omega$, the effects of magnetic diffusion (diffusivity $\eta$ ), and the reduction of buoyancy by thermal diffusion on small scales (diffusivity $\kappa$ ).

First we checked the analytic prediction of which azimuthal orders $m$ should be unstable, depending on the value of $p$ (Eq. (4)). We have confirmed this prediction (see Sect. 4.1) for

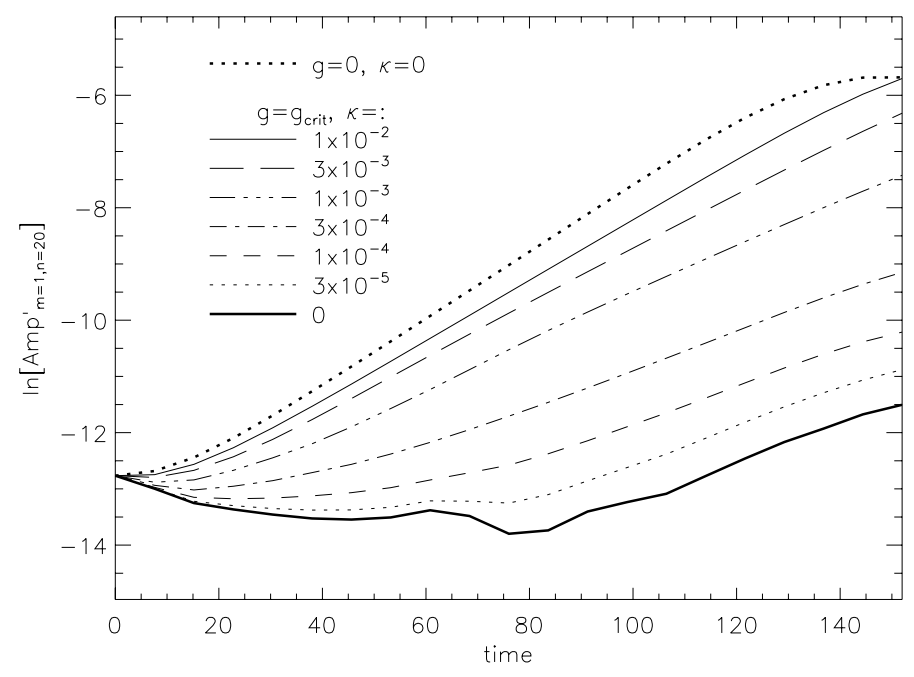

Fig. 15. Velocity field amplitudes of the $m=1, n=20$ mode as a function of time, at different values of thermal diffusion $\kappa$, when $g=$ $10 / \pi$. For large values of $\kappa$, the stabilising effect of gravity is absent and growth occurs at the same rate as in the adiabatic unstratified case (uppermost line).

the cases $p=1$ and $p=2$, the former being considered the most important as it is this magnetic field which could plausibly be produced by the winding-up of a seed field by differential rotation. The dominant mode has $m=1$, a "kink" instability.

The theory predicts that in the adiabatic, unstratified case ( $\kappa=\eta=N=0$ ) there is no threshold for instability, and that the growth rate $\sigma$ is of the order of the angular frequency $\omega_{\mathrm{A}}=r / v_{\mathrm{A}}$ of an Alfvén wave travelling around the star on an azimuthal field line. The field strength and hence $\omega_{\mathrm{A}}$ varies through the computational volume, but since the instability is a local one, the growth should be dominated by the largest value of $\omega_{\mathrm{A}}$ in the volume, after an initial transient. The numerical results reproduce this well. In the best studied case, for example, a value $\sigma / \omega_{\mathrm{A}}=0.92$ was measured.

In the adiabatic case the prediction (Pitts \& Tayler 1986) is that the instability is suppressed when the rotation rate exceeds the Alfvén frequency $\omega_{\mathrm{A}}$. This was also verified, give or take a few percent at the most. The adiabatic case is somewhat singular with respect to the effect of rotation, however. Theory predicts that in the presence of strong thermal diffusion $(\kappa / \eta \rightarrow \infty)$ the threshold for instability disappears again, and that the growth rate is then of the order $\sigma=\omega_{\mathrm{A}}^{2} / \Omega$. We have not tested this dependence, since the calculations required for this limiting case are computationally rather demanding.

The effect of gravity and both magnetic and thermal diffusion were investigated. The effect of gravity was as expected - above a certain value of $g$ the initial equilibrium is stable at a given vertical wavenumber $n$. This translates into a minimum unstable wavenumber which increases with increasing $g$.

The effect of magnetic diffusion on the shorter wavelengths was not exactly the same as that expected. It was found that at a given wavenumber $n$, a value of $\eta$ of the order suggested by Acheson (1978) and Spruit (1999) did not kill the instability entirely, rather it reduced its growth rate by about half. An increase in $\eta$ beyond this reduced the growth rate still further, but not to zero. Therefore it seems that magnetic diffusion alone cannot suppress the instability. It is possible that the discrepancy arises because the case $\eta \rightarrow \infty$ may have a singular limit. The theoretical prediction was made for the case $\kappa=0$, but the relevant parameter is actually $\kappa / \eta$. By analogy with other double-diffusive systems, instabilities must exist also in the case $\kappa<\eta$ which 
do not appear when $\kappa=0$ is set from the beginning. Since this case $\kappa<\eta$ is not of much astrophysical relevance, we have not pursued this further.

Finally, the effect of thermal diffusion was tested. In the runs executed, it was expected that values of $\kappa$ of the order of $9 \times 10^{-5}$ and $2.7 \times 10^{-4}$ would be needed to make a run with $g=g_{\text {crit }}$ behave like the runs with $g=0.8 g_{\text {crit }}$ and $g=0.6 g_{\text {crit }}$ respectively. This appears to be correct, given that these were only order-ofmagnitude approximations.

The non-linear effect of the instability in all the above cases was found to be rather simple. The net effect is similar to that of an enhanced magnetic diffusivity: the field configuration spreads horizontally, while the mean azimuthal magnetic flux decreases due to effective reconnection across the magnetic axis. Toroidal fields in stars are therefore predicted to decay quickly by Tayler instability, once conditions for instability are satisfied, unless regenerated by differential rotation. Work continues on the nonlinear evolution of this instability, and some first results can be found in Braithwaite (2006).

\section{References}

Acheson, D. J. 1978, Phil. Trans. Roy. Soc. Lond. A, 289, 459

Balbus, S. A., \& Hawley, J. F. 1992, ApJ, 400, 610

Braithwaite, J. 2006, A\&A, 449, 451
Braithwaite, J., \& Spruit, H. C. 2004, Nature, 431, 891

Chaplin, W. J., Elsworth, Y., Isaak, G. R., et al. 2001, MNRAS, 327, 1127

Cowling, T. G. 1945, MNRAS, 105, 166

Gilman, P. A. 1970, ApJ, 162, 1019

Goossens, M., Biront, D., \& Tayler, R. J. 1981, Ap. Space Sci., 75, 521

Hawley, J. F., Gammie, C. F., \& Balbus, S. A. 1995, ApJ, 440, 742

Heger, A., Langer, N., \& Woosley, S. E. 2000, ApJ, 528, 368

Heger, A., Woosley, S. E., Langer, N., \& Spruit, H. C. 2003, Proc. IAU Symp., 215

Hyman, J. 1979, in Adv. in Comp. Meth. for PDEs - III, ed. R. Vichnevetsky, \& R. S. Stepleman, 313

Maeder, A., \& Meynet, G. 2003, A\&A, in press

Menou, K., Balbus, S. A., \& Spruit, H. C. 2004, ApJ, 607, 564

Mestel, L. 1953, MNRAS, 113, 716

Nordlund, Å., \& Galsgaard, K. 1995, http://www. astro.ku.dk/ aake/papers/95.ps.gz

Paczyński, B. 1998, ApJ, 494, 45

Parker, E. N. 1955, ApJ, 121, 491

Pitts, E., \& Tayler, R. J. 1986, MNRAS, 216, 139

Spruit, H. C. 1998, A\&A, 333, 603

Spruit, H. C. 1999, A\&A, 349, 189

Spruit, H. C. 2002, A\&A, 381, 923

Spruit, H. C., \& Phinney, E. S. 1998, Nature, 393, 139

Tayler, R. J. 1957, Proc. Phys. Soc. B, 70, 311957

Tayler, R. J. 1973, MNRAS, 161, 365

Velikhov, E. P. 1959, J. Exp. Theoret. Phys. (USSR), 36, 1398

Woosley, S. E. 1993, ApJ, 405, 273

Zahn, J.-P. 1974, in Stellar Instability and Evolution, IAU Symp., 59, 185

Zahn, J.-P. 1983, in Astrophysical Processes in Upper Main Sequence Stars, ed. A. Maeder, Geneva Observatory, 253 